\title{
A Damped Ly $\alpha$ Absorption-Line System in an Apparent Void at Redshift 2.38
}

\author{
Leith E. H. Godfrey ${ }^{\mathrm{A}}$ and Paul J. Francis ${ }^{\mathrm{A}, \mathrm{B}}$ \\ A Research School of Astronomy and Astrophysics, Australian National University, \\ Canberra ACT 0200, Australia \\ B Corresponding author. Email: pfrancis@mso.anu.edu.au
}

Received 2005 February 16, accepted 2005 June 22

\begin{abstract}
We study the contents of an apparent void in the distribution of Ly $\alpha$ emitting galaxies at redshift 2.38. We show that this void is not empty, but contains a damped Ly $\alpha$ absorption-line system, seen in absorption against background QSO 2138-4427. Imaging does not reveal any galaxy associated with this absorption-line system, but it contains metals $(\mathrm{Fe} / \mathrm{H} \sim-1.3)$, and its large velocity range $\left(\sim 180 \mathrm{~km} \mathrm{~s}^{-1}\right)$ implies a significant mass.
\end{abstract}

Keywords: Large Scale Structure of Universe — quasars: individual (2138-4427) — quasars: absorption lines

\section{Introduction}

It is now abundantly clear that high redshift galaxies are as strongly clustered as those in the local universe (e.g. Giavalisco et al. 1998; Steidel et al. 2000). The topology of this clustering is not as clear, but there is some evidence that high redshift galaxies, just like their local descendants, lie in filamentary structures, separated by voids (e.g. Campos et al. 1999; Møller \& Fynbo 2001; Palunas et al. 2004; Turnshek et al. 2004).

In the local universe, voids are surprisingly free of galaxies (e.g. Peebles 2001). The few galaxies seen in voids are unusually blue and tend to be centrally concentrated (e.g. Roias et al. 2004, and references therein). Low column density Ly $\alpha$ forest QSO absorption-line systems can also be found in voids (Shull, Stocke, \& Penton 1996; Penton, Stocke, \& Shull 2004). At higher redshifts, cold dark matter modelling predicts that galaxy biassing should be much stronger than today (e.g. Fry 1996; Bagla 1998; Tegmark \& Peebles 1998; Baugh et al. 1999). The dark matter underdensity in voids should thus be much less than it is today. One might thus expect to find more or different objects in any voids.

Palunas et al. (2004) mapped an $80 \times 60 \times 60$ comoving Mpc region of the high redshift universe, at $z=2.38$ (see also Francis et al. 2004). They showed that the distribution of Ly $\alpha$ emitting galaxies was not random. The void probability function was significantly higher than that expected for randomly distributed galaxies on scales of 5-10 Mpc. The signal was dominated by a single large empty region just to the north-west of their field centre.

These voids are deficient in Ly $\alpha$ emitting galaxies, but do they contain anything else? Luckily, a luminous background QSO, LBQS 2138-4427, lies behind the major void. Francis \& Hewett (1993) showed that this QSO had a damped Ly $\alpha$ absorption-line system at redshift 2.383. This redshift places the damped system clearly within the major Palunas et al. void, more than 10 co-moving Mpc from any Ly $\alpha$ emitting galaxies.

Is the line of sight to this QSO really passing through a void, or is the lack of Ly $\alpha$ emitting galaxies close to it an artefact of small number statistics? In Palunas et al. (2004) we generated mock galaxy catalogues with the same total number of Ly $\alpha$ emitting galaxies as were observed. These mock catalogues assumed a random distribution of galaxies, but allowed for differences in exposure time across our field (the QSO is in a region with the above average exposure time). Less than $1 \%$ of these random catalogues had no Ly $\alpha$ emitting galaxies within 10 projected co-moving Mpc of the QSO sight line. We therefore tentatively conclude that this sight line is probing a region that is underdense in Ly $\alpha$ emitting galaxies.

In this paper, we show that imaging fails to identify any galaxy candidate that could be associated with this damped Ly $\alpha$ absorption-line system. We present highresolution spectroscopy which demonstrates, however, that the damped system is substantially enriched with a wide variety of heavy elements. The velocity sub-structure of the damped system also indicates the likely presence of significant mass. This void is thus far from empty.

\section{Imaging}

The area around QSO 2138-4427 was imaged by Palunas et al. (2004), down to a Ly $\alpha$ flux limit of $1.4 \times 10^{-16} \mathrm{erg} \mathrm{cm}^{-2} \mathrm{~s}^{-1}$, and down to (AB) broad-band magnitude limits of $B=26.2, V=25.3, R=24.0$, and $I=23.8$. Nothing was seen within $10 \operatorname{arcsec}$ of the QSO in any of these bands: it is an unusually clear sight line. Near-IR imaging was obtained on the nights of 
1998 September 6-7, using the CASPIR (Cryogenic Array Spectrometer/Imager) camera on the Siding Spring 2.3-m telescope. A total of $4.5 \mathrm{~h}$ exposure time was obtained in the H-band, reaching a (Vega) magnitude limit of $H=20.5$. Once again nothing was seen within $10 \operatorname{arcsec}$ of the QSO.

\section{Spectroscopy}

\subsection{Observations and Reduction}

Our existing spectra of LBQS 2138-4427 (Francis \& Hewett 1993) were of too low a resolution and narrow a wavelength coverage to seriously constrain the physical properties of the gas. We therefore re-observed it using the University College London Echelle Spectrograph (UCLES) on the Anglo-Australian Telescope (AAT). Observations were carried out on the nights of 2001 August 20-23 and the total usable integration time was 43200 s. D'Odorico, Petitjean, \& Cristiani (2002) independently obtained high-resolution spectra of this QSO, using the VLT Ultraviolet Visual Echelle Spectrograph (UVES). They kindly allowed us to use their spectrum in this analysis. Both spectra have velocity resolutions corresponding to a Doppler parameter $b=6 \mathrm{~km} \mathrm{~s}^{-1}$. Where they overlap, the two spectra agree very well.

The data were reduced using standard procedures, and set to the vacuum heliocentric frame. The UVES spectrum has a higher signal-to-noise ratio, and a wider wavelength coverage in the red: it was used for our analysis of most lines. The CIV doublet, however, was not covered by UVES, so we used the UCLES spectrum.

Absorption-line properties were measured interactively using the XvOIGT program (Mar \& Bailey 1995). Upper and lower limits were derived interactively by varying parameters until residuals clearly exceeded the noise. In most cases, possible line blending and/or saturation dominated noise as the major source of uncertainty.

\subsection{Results}

The damped Ly $\alpha$ system was clearly detected in a wide variety of transitions (Figure 1). Low ionization transitions such as OI and MgII dominated, though a relatively weak detection was made in CIV.

In the majority of transitions, the line breaks up into about nine sub-components, with relative velocities spanning $178 \mathrm{~km} \mathrm{~s}^{-1}$. Each individual component is spectrally unresolved, or at best marginally resolved. The measured properties of the components are shown in Table 1. The neutral hydrogen content of each individual component cannot be constrained, though the total neutral hydrogen content of the system is $\log (\mathrm{N}(\mathrm{HI}))=20.5 \pm 0.1$. In some cases (CII, MgII), the inner components blended into a single absorption feature, and consequently an estimate of the column density for each inner component was not possible. However, an upper limit on the combined column density could be determined in these cases. Several ionic transitions did not produce observable absorption features (either due to blending with unassociated lines or to low column density). For these ions, only upper limits on the column density were possible (assuming that their velocity structure was the same as that of the other transitions). CIV clearly has a different profile from the other (lower ionization) transitions and was best fit with two components at $v_{\text {rel }}=-2$ and $14 \mathrm{~km} \mathrm{~s}^{-1}$, each having $\mathrm{N}$ (CIV) $\sim 10^{14.2} \mathrm{~cm}^{-2}$ with velocity dispersion $b \sim 10-20 \mathrm{~km} \mathrm{~s}^{-1}$.

\subsection{Composition and Physical Properties of the Gas}

This system appears to be a very typical damped Lya system. The predominance of low ionization species and the narrowness of the individual sub-components suggest that they are cool and relatively dense. We modelled a typical low ionization sub-component, using Gary Ferland's CLOUDY code (Ferland 1996), and assuming photoionization of a uniform density, plane parallel slab by a typical diffuse UV background (Haardt \& Madau 1996; Scott et al. 2000; Simcoe, Sargent, \& Rauch 2004). The line ratios are consistent with the hydrogen being predominantly neutral (Dessauges-Zavadsky et al. 2004), though we are unable to completely rule out partial hydrogen ionization (e.g. Prochaska et al. 2002; Vladilo et al. 2001; Izotov, Schaerer, \& Charbonnel 2001). The modelling suggests densities of $\sim 10^{-3}-10^{-1} \mathrm{~cm}^{-3}$ and a temperature of $\sim 10^{4} \mathrm{~K}$. If we assume that the gas in these components is mostly neutral, we infer mean metallicities $[\mathrm{Fe} / \mathrm{H}],[\mathrm{Si} / \mathrm{H}]$, $[\mathrm{O} / \mathrm{H}]$, and $[\mathrm{Al} / \mathrm{H}]$ all of $\sim-1.3$. This is compared with the mean metallicity of $\langle[\mathrm{Zn} / \mathrm{H}]\rangle \approx-1.15 \pm 0.15$ dex for DLAs at $z>1.5$ (Prochaska \& Wolfe 1999).

\section{Conclusion}

We conclude that this apparent void is not empty: it contains a very typical damped Ly $\alpha$ absorption-line system. The non-detection of any galaxy counterpart in our imaging is very consistent with this: only a tiny fraction of damped systems have identifiable counterparts (e.g. Bouché \& Lowenthal 2004, and references therein). The galaxy counterparts presumably either lie under the pointspread function of the background QSO or are below the detection limits.

The absorption-line spectra clearly show, however, that the gas in the damped system is condensed into cool cloudlets, and that it is enriched in a wide variety of elements. The large velocity dispersion between the components further suggests that quite a large mass may be present.

We are not implying that all damped Ly $\alpha$ systems arise in voids. The inter-relationship of damped systems and galaxies is clearly complex. On large scales, Lyman-break galaxies and damped systems seem, on average, to trace each other (Bouché \& Lowenthal 2004). In our field, there are two additional damped Ly $\alpha$ absorption-line systems seen in the spectra of two different background QSOs. One of these lies within an apparent proto-cluster (Francis \& Williger 2004), while the other lies on the fringe of the major void (Francis, Wilson, \& Woodgate 2001). To add to the complexity, we are delineating the void using Ly $\alpha$ emitting galaxies. Due to its high optical depth and easy 


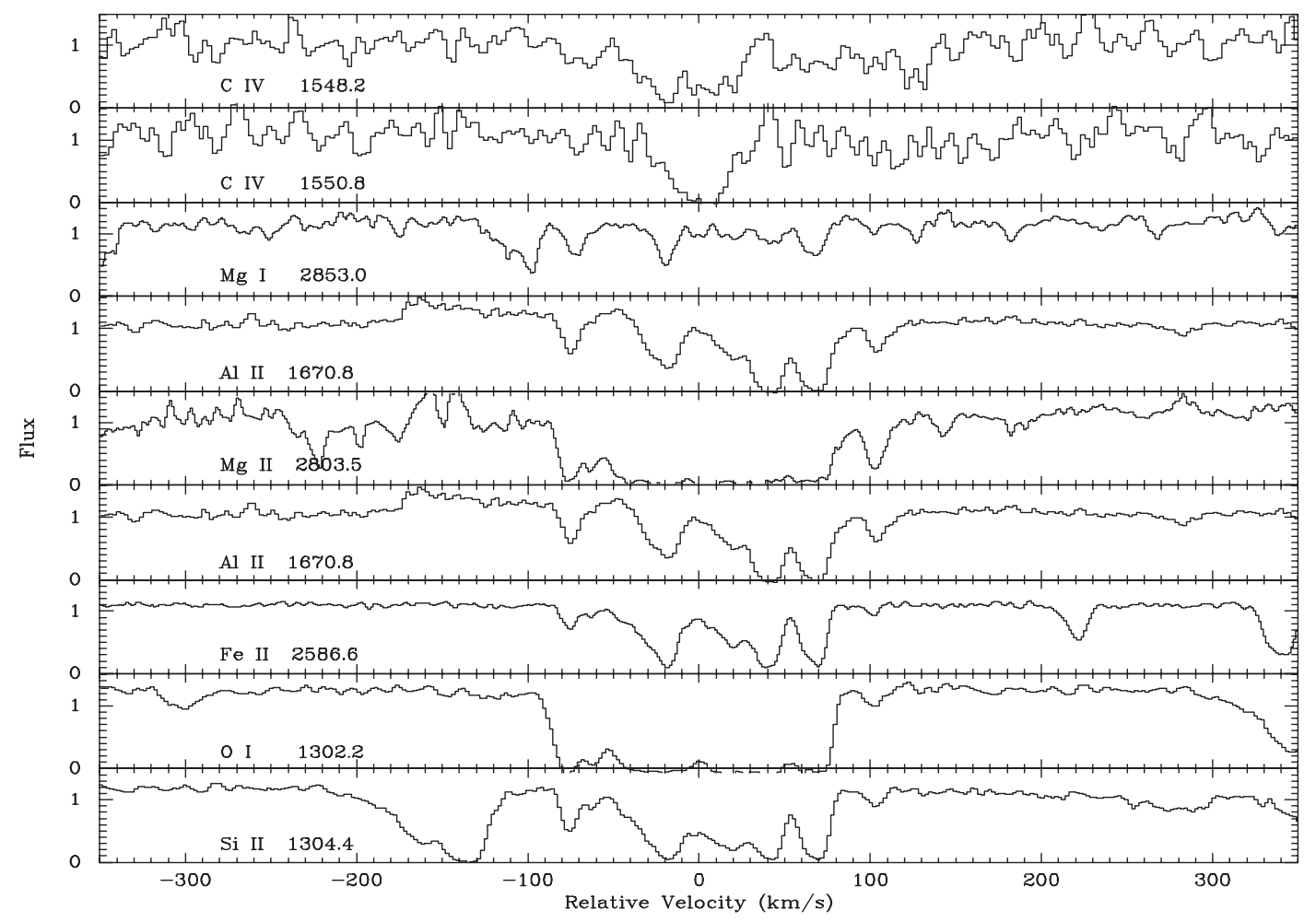

Figure 1 Spectra of the nine different transitions. The CIV data are from the UCLES spectrum, and the others are from the UVES spectrum. Velocities are relative to a nominal redshift of 2.383 .

Table 1. Measured parameters of low-ionization sub-components

\begin{tabular}{|c|c|c|c|c|c|c|c|c|c|}
\hline \multirow[t]{2}{*}{ Property } & \multicolumn{9}{|c|}{ Sub-component } \\
\hline & 1 & 2 & 3 & 4 & 5 & 6 & 7 & 8 & 9 \\
\hline$v_{\text {rel }}{ }^{\mathrm{a}}\left(\mathrm{km} \mathrm{s}^{-1}\right)$ & -74 & -59 & -42 & -37 & -16 & 21 & 43 & 68 & 104 \\
\hline \multirow[t]{2}{*}{$b^{\mathrm{b}}\left(\mathrm{km} \mathrm{s}^{-1}\right)$} & $<6$ & $<6$ & $<7$ & $<8$ & $<10$ & $<12$ & $<10$ & $<9$ & $<6$ \\
\hline & \multicolumn{9}{|c|}{$\log ($ column density $)\left(\mathrm{cm}^{-2}\right)$} \\
\hline $\mathrm{CI}$ & $<12.6$ & $<12.7$ & $<12.7$ & $<12.7$ & $<12.7$ & $<12.7$ & $<12.7$ & $<12.7$ & $<12.7$ \\
\hline CII & $14.4_{-0.4}^{+0.9}$ & $14.2_{-0.6}^{+0.6}$ & $\ldots$ & $\ldots$ & $\ldots$ & $\ldots$ & $\ldots$ & $\ldots$ & $13.8_{-0.2}^{+1.2}$ \\
\hline NI & $<13.3^{-0.4}$ & $<13.7$ & $<13.5$ & $<13.7$ & $<14.5$ & $<14.1$ & $<14.5$ & $<14.0$ & $<13.6$ \\
\hline OI & $15.0_{-0.5}^{+1.9}$ & $14.4_{-0.2}^{+1.6}$ & $14.2_{-0.2}^{+1.7}$ & $14.6_{-0.4}^{+3.0}$ & $14.9_{-0.1}^{+1.0}$ & $15.0_{-0.2}^{+1.8}$ & $15.2_{-0.6}^{+1.8}$ & $15.6_{-0.6}^{+1.3}$ & $13.1_{-0.3}^{+0.3}$ \\
\hline MgI & $11.6_{\mathrm{inf}}^{+0.3}$ & $11.1_{\mathrm{inf}}^{+0.3}$ & $<11.2$ & $<11.2$ & $12.0_{-0.2}^{+0.2}$ & $11.5_{-0.2}^{+0.3}$ & $11.6_{-0.2}^{+0.3}$ & $11.9_{-0.2}^{+0.2}$ & $11.4_{-0.6}^{+0.4}$ \\
\hline MgII & $13.4_{-0.5}^{+0.8}$ & $13.0_{-0.3}^{+0.5}$ & $\ldots$ & $\ldots$ & $\ldots$ & $\ldots$ & $\ldots$ & $\ldots$ & $13.1_{-0.3}^{+0.6}$ \\
\hline AliII & $12.1_{-0.2}^{+0.4}$ & $11.5_{-0.5}^{+0.2}$ & $11.0_{-1.0}^{+0.5}$ & $11.9_{-0.3}^{+0.5}$ & $12.4_{-0.1}^{+0.2}$ & $12.4_{-0.2}^{+0.3}$ & $13.4_{-0.5}^{+1.6}$ & $13.3_{-0.5}^{+1.6}$ & $12.0_{-0.2}^{+1.1}$ \\
\hline SiI & $<12.0$ & $<12.0$ & $<12.0$ & $<12.0$ & $<12.0$ & $<12.0$ & $<12.0$ & $<12.0$ & $<12.0$ \\
\hline SiII & $13.9_{-0.6}^{+1.6}$ & $12.8_{-0.2}^{+0.8}$ & $13.0_{-0.3}^{+0.2}$ & $13.0_{-0.3}^{+0.4}$ & $14.0_{-0.1}^{+0.2}$ & $14.0_{-0.1}^{+0.2}$ & $14.4_{-0.6}^{+0.8}$ & $13.9_{-0.4}^{+0.3}$ & $13.1_{-0.1}^{+0.2}$ \\
\hline SiIII & $<12.6$ & $<12.3$ & $<12.7$ & $<12.5$ & $<15.4$ & $<14.8$ & $<13.0$ & $<16.3$ & $<13.6$ \\
\hline Sirv & $<12.4$ & $<12.4$ & $<12.2$ & $<12.3$ & $<12.4$ & $<12.4$ & $<12.2$ & $<12.2$ & $<12.5$ \\
\hline SI & $<12.7$ & $<12.7$ & $<12.7$ & $<12.7$ & $<12.7$ & $<12.7$ & $<12.7$ & $<12.7$ & $<12.7$ \\
\hline CrII & $<12.0$ & $<12.0$ & $<12.0$ & $<12.1$ & $<12.6$ & $<12.1$ & $<12.5$ & $<12.4$ & $<12.2$ \\
\hline FeII & $13.2_{-0.2}^{+0.1}$ & $12.9_{-0.3}^{+0.1}$ & $12.9_{-0.2}^{+0.4}$ & $13.2_{-0.2}^{+0.4}$ & $14.2_{-0.4}^{+1.6}$ & $13.7_{-0.2}^{+0.3}$ & $14.0_{-0.1}^{+2.3}$ & $14.0_{-0.1}^{+0.6}$ & $12.4_{-0.1}^{+0.3}$ \\
\hline FeIII & $<14.0$ & $<13.5$ & $<13.9$ & $<14.3$ & $<14.1$ & $<14.1$ & $<14.1$ & $<13.8$ & $<13.0$ \\
\hline NiII & $<12.6$ & $<12.6$ & $<12.6$ & $<12.6$ & $<13.0$ & $<12.4$ & $<12.8$ & $<12.6$ & $<12.6$ \\
\hline ZnII & $<11.3$ & $<11.1$ & $<11.2$ & $<11.2$ & $<11.6$ & $<11.5$ & $<11.5$ & $<11.4$ & $<11.4$ \\
\hline
\end{tabular}

a Velocity of component relative to $z=2.383$.

${ }^{\mathrm{b}}$ Velocity dispersion of individual sub-component. 
destruction by dust, Ly $\alpha$ emission is not expected to correlate straightforwardly with any easily modellable property of a galaxy, such as its mass or star formation rate - their distribution is thus hard to model. In addition, the apparent void is only $99 \%$ significant.

We tentatively conclude, however, that at least one damped system lies within an apparent void, and that this damped system contains significant metal enrichment, and is probably associated with considerable mass.

\section{Acknowledgment}

We wish to thank Cedric Ledoux for making the UVES spectrum available to us.

\section{References}

Bagla, J. S. 1998, MNRAS, 299, 417

Baugh, C. M., Benson, A. J., Cole, S., Frenck, C. S., \& Lacey, C. G. 1999, MNRAS, 305, L21

Bouché, N., \& Lowenthal, J. D. 2004, ApJ, 609, 513

Campos, A., Yahil, A., Windhorst, R. A., Richards, E. A., Pascarelle, S., Impey, C., \& Petry, C. 1999, ApJL, 511, L4

D’Odorico, V., Petitjean, P., \& Cristiani, S. 2002, A\&A, 390, 13

Dessauges-Zavadsky, M., Calura, F., Prochaska, J. X., D’Odorico, S., \& Matteucci, F. 2004, A\&A, 416, 79

Ferland, G. J. 1996, HAZY, a Brief Introduction to CLOUDY, University of Kentucky Department of Physics and Astronomy Internal Report

Francis, P. J., \& Hewett, P. C. 1993, AJ, 106, 2587
Francis, P. J., Palunas, P., Teplitz, H. I., Williger, G. M., \& Woodgate, B. E. 2004, ApJ, 614, 75

Francis, P. J., \& Williger, G. M. 2004, ApJ, 602, 77

Francis, P. J., Wilson, G. M., \& Woodgate, B. E. 2001, PASA, 18, 64

Fry, J. N. 1996, ApJ, 461, 65

Giavalisco, M., Steidel, C. C., Adelberger, K. L., Dickinson, M. E., Pettini, M., \& Kellogg, M. 1998, ApJ, 503, 543

Haardt, F., \& Madau, P. 1996, ApJ, 461, 20

Izotov, Y. I., Schaerer, D., \& Charbonnel, C. 2001, ApJ, 549, 878

Mar, D. P., \& Bailey, J. G. 1995, PASA, 12, 239

Møller, P., \& Fynbo, J. U. 2001, A\&A, 372, L57

Palunas, P., Teplitz, H. I., Francis, P. J., Williger, G. M., \& Woodgate, B. E. 2004, ApJ, 602, 545

Peebles, P. J. E. 2001, ApJ, 557, 495

Penton, S. V., Stocke, J. T., \& Shull, J. M. 2004, ApJS, 152, 29

Prochaska, J. X., Howk, J. C., O’Meara, J. M., Tytler, D., Wolfe, A. M., Kirkman, D., Lubin, D., \& Suzuki, N. 2002, ApJ, 571, 693

Prochaska, J. X., \& Wolfe, A. M. 1999, ApJ, 121, 369

Roias, R. R., Vogeley, M. S., Hoyle, F., \& Brinkmann, J. 2004, ApJ, 617,50

Scott, J., Bechtold, J., Dobrzycki, A., \& Kulkarni, V. P. 2000, ApJS, 130,67

Shull, M. J., Stocke, J. T., \& Penton, S. 1996, AJ, 111, 72

Simcoe, R. A., Sargent, W. L. W., \& Rauch, M. 2004, ApJ, 606, 92

Steidel, C. C., Adelberger, K. L., Shapley, A. E., Pettini, M., Dickinson, M., \& Giavalisco, M. 2000, ApJ, 532, 170

Tegmark, M., \& Peebles, P. J. E. 1998, ApJL, 500, L79

Turnshek, D. A., Rao, S. M., Nestor, D. B., Vanden Berk, D., BelfortMihalyi, M., \& Monier, E. M. 2004, ApJL, 609, L53

Vladilo, G., Centurión, M., Bonifacio, P., \& Howk, J. C. 2001, ApJ, 557, 1007 